\title{
Quantification of interfacial spin-charge conversion in hybrid devices with a metal/ insulator interface
}

Cite as: Appl. Phys. Lett. 117, 142405 (2020); https://doi.org/10.1063/5.0023992

Submitted: 03 August 2020 . Accepted: 11 September 2020. Published Online: 06 October 2020

(D) Cristina Sanz-Fernández, (D) Van Tuong Pham, Edurne Sagasta, (D) Luis E. Hueso, (D) llya V. Tokatly, (D) Fèlix Casanova, and F. Sebastián Bergeret

ARTICLES YOU MAY BE INTERESTED IN

Field free magnetization switching in perpendicularly magnetized $\mathrm{Pt} / \mathrm{Co} / \mathrm{FeNi} / \mathrm{Ta}$ structure by spin orbit torque

Applied Physics Letters 117, 142404 (2020); https://doi.org/10.1063/5.0025132

Robust spin-orbit torques in ferromagnetic multilayers with weak bulk spin Hall effect Applied Physics Letters 117, 122401 (2020); https://doi.org/10.1063/5.0011399

Pure spin current phenomena

Applied Physics Letters 117, 190501 (2020); https://doi.org/10.1063/5.0032368

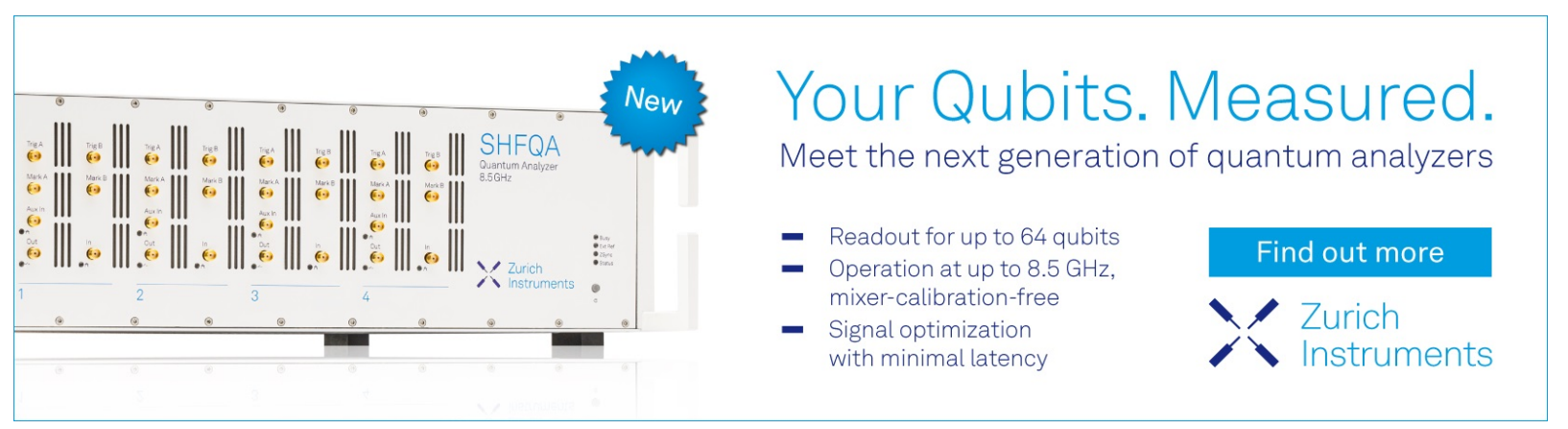




\title{
Quantification of interfacial spin-charge conversion in hybrid devices with a metal/insulator interface
}

Cite as: Appl. Phys. Lett. 117, 142405 (2020); doi: 10.1063/5.0023992

Submitted: 3 August 2020 - Accepted: 11 September 2020 .

Published Online: 6 October 2020

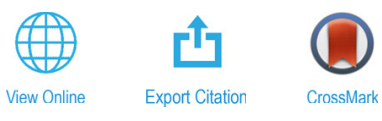

Cristina Sanz-Fernández, ${ }^{1, a)}$ iD Van Tuong Pham, ${ }^{2, a}$ Fèlix Casanova, ${ }^{2,3, a)}$ (D) and F. Sebastián Bergeret ${ }^{1,5, a)}$ (iD

\author{
AFFILIATIONS \\ ${ }^{7}$ Centro de Física de Materiales (CFM-MPC), Centro Mixto CSIC-UPV/EHU, 20018 Donostia-San Sebastián, Basque Country, Spain \\ ${ }^{2} \mathrm{CIC}$ nanoGUNE, 20018 Donostia-San Sebastián, Basque Country, Spain \\ ${ }^{3}$ IKERBASQUE, Basque Foundation for Science, 48013 Bilbao, Basque Country, Spain \\ ${ }^{4}$ Nano-Bio Spectroscopy Group, Departamento de Física de Materiales, Universidad del País Vasco (UPV/EHU), \\ 20018 Donostia-San Sebastián, Basque Country, Spain \\ ${ }^{5}$ Donostia International Physics Center (DIPC), 20018 Donostia-San Sebastián, Basque Country, Spain
}

a) Authors to whom correspondence should be addressed: cristina_sanzO01@ehu.eus; v.pham@nanogune.eu; ilya.tokatly@ehu.es; f.casanova@nanogune.eu; and fs.bergeret@csic.es

\begin{abstract}
We present and experimentally verify a universal theoretical framework for the description of spin-charge interconversion in non-magnetic metal/insulator structures with interfacial spin-orbit coupling (ISOC). Our formulation is based on drift-diffusion equations supplemented with generalized boundary conditions. The latter encode the effects of ISOC and relate the electronic transport in such systems to spin loss and spin-charge interconversion at the interface. We demonstrate that the conversion efficiency depends solely on these interfacial parameters. We apply our formalism to two typical spintronic devices that exploit ISOC: a lateral spin valve and a multilayer Hall bar, for which we calculate the non-local resistance and the spin Hall magnetoresistance, respectively. Finally, we perform measurements on these two devices with a $\mathrm{BiO}_{x} / \mathrm{Cu}$ interface and verify that transport properties related to the ISOC are quantified by the same set of interfacial parameters.
\end{abstract}

Published under license by AIP Publishing. https://doi.org/10.1063/5.0023992

A thorough understanding of charge and spin transport in systems with spin-orbit coupling (SOC) is crucial for the electric control of spin currents. ${ }^{1,2}$ The latter leads to the widely studied spin Hall effect $(\mathrm{SHE})^{3-5}$ and Edelstein (EE) effect, ${ }^{6-9}$ which are at the basis of spin-orbit torque memories ${ }^{10-12}$ and spin-based logic devices. ${ }^{13,14}$

Of particular interest are systems with spin-charge interconversion (SCI) at the interface between an insulator (I) with a heavy element and a normal metal (N) with negligible SOC and long spin relaxation length as, for example, $\mathrm{BiO}_{x} / \mathrm{Cu}$ bilayers. In these systems, the SCI occurs at the hybrid interface via an interfacial spin-orbit coupling (ISOC). ${ }^{15,16}$ Whereas the electronic transport in $\mathrm{N}$ is well described by customary drift-diffusion equations, the interfacial effects occur at atomic scales near the interface and, hence, their inclusion is more subtle. Some works use an intuitive picture based on an idealized 2DEG with Rashba SOC at the interface, ${ }^{16-18}$ in which the interconversion takes place via the $\mathrm{EE}$ and its inverse (IEE). Such a description is clearly valid for conductive surface states in (e.g., topological) insulators $^{19,20}$ or 2DEGs. ${ }^{21,22}$ However, in metallic systems, it requires additional microscopic parameters to model the coupling between interface states and the diffusive motion of electrons in the metal. Moreover, the very existence of a well-defined two-dimensional interface band and its relevance for the electronic transport in systems such as $\mathrm{BiO}_{x} / \mathrm{Cu}$ is not obvious as realistic structures are frequently polycrystalline and disordered. Moreover, one can contemplate other microscopic scenarios to describe the SCI. For example, at the $\mathrm{BiO}_{x} / \mathrm{Cu}$ interface, $\mathrm{Bi}$ atoms could diffuse into $\mathrm{Cu}$ inducing an effective extrinsic SHE in a thin layer near the interface. ${ }^{23}$ Alternatively, a SCI can be generated via an interfacial spin-dependent scattering of the bulk Bloch states. ${ }^{24-26}$ Each of these scenarios will invoke different sets of microscopic parameters to be inferred from macroscopic transport measurements.

In this Letter, we approach the problem from a different angle and propose a universal theoretical framework, which is independent 
of microscopic details. We combine the drift-diffusion theory with effective boundary conditions (BCs) ${ }^{27}$ to account for ISOC. Such BCs describe two types of interfacial processes: SCI and spin-losses, quantified, respectively, by the interfacial spin-to-charge/charge-to-spin conductivities, $\sigma_{\text {sc } / \mathrm{cs}}$, and the spin-loss conductances $G_{\| / \perp}$ for spins polarized parallel/perpendicular to the interface. The SCI efficiency is determined by the ratio between the strengths of these two processes. This ratio coincides with the widely used conversion efficiency and the inverse Edelstein length $\lambda_{\mathrm{IEE}}$ such that $\lambda_{\mathrm{IEE}}=\sigma_{\mathrm{sc}} / G_{\|}$. Furthermore, we apply our theory to describe two typical experimental setups: non-local resistance measurement in a Permalloy/copper $(\mathrm{Py} / \mathrm{Cu})$ lateral spin valve (LSV) with a middle $\mathrm{BiO}_{x} / \mathrm{Cu}$ wire, Fig. 2(a), and measurement of the spin Hall magnetoresistance (SMR) in a $\mathrm{BiO}_{x} / \mathrm{Cu} / \mathrm{YIG}$ trilayer Hall bar, Fig. 4(a). From the fitting of our theory to the experimental results, we show that both experiments are described by similar values of the ISOC parameters. This confirms that the SCI only depends on the intrinsic properties of the $\mathrm{BiO}_{x} / \mathrm{Cu}$ interface. Moreover, we demonstrate that $\sigma_{\mathrm{sc}}=\sigma_{\mathrm{cs}}$, in accordance with Onsager reciprocity.

We start considering the I/N structure depicted in Fig. 1. In the $\mathrm{N}$ layer, spin and charge transport is described by the diffusion equations,

$$
\begin{gathered}
\nabla^{2} \hat{\mu}=\frac{\hat{\mu}}{\lambda_{\mathrm{N}}^{2}}, \\
\nabla^{2} \mu=0 .
\end{gathered}
$$

Here, $\hat{\mu}=\left(\mu^{x}, \mu^{y}, \mu^{z}\right)$ and $\mu$ are the spin and charge electrochemical

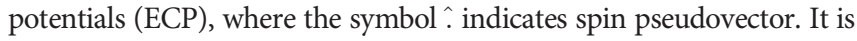

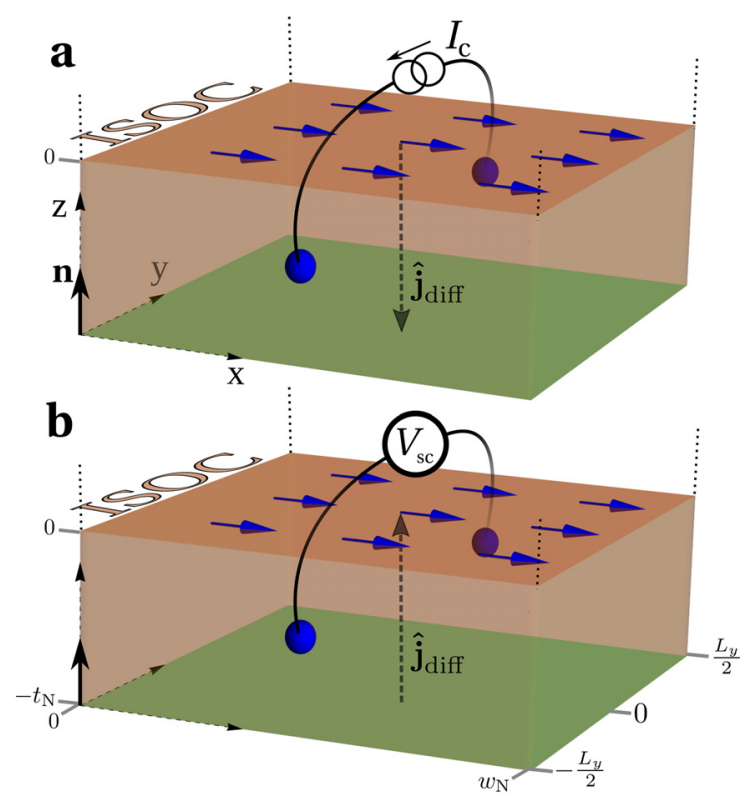

FIG. 1. Sketch of the non-magnetic insulator $(z>0) /$ metal $(z<0)$ system under study. ISOC is finite at the interface with normal vector $\mathbf{n}$. (a) Charge-to-spin conversion: a charge current $I_{c}$ induces at the interface an out-of-plane spin current density $\hat{\mathbf{j}}_{\text {diff }}$ perpendicularly polarized. (b) Spin-to-charge conversion: a $\mathbf{n}$ injected spin current density $\hat{\mathbf{j}}_{\text {diff }}$ induces at the interface a voltage drop perpendicular to the polarization of $\hat{\mathbf{j}}_{\text {diff }}$. assumed that $\mathrm{N}$ has inversion symmetry with an isotropic spin relaxation described by the spin diffusion length $\lambda_{\mathrm{N}}{ }^{28}$ The diffusive charge and spin currents are defined as $e \hat{\mathbf{j}}=-\sigma_{\mathrm{N}} \nabla \hat{\mu}$ and $e \mathbf{j}=-\sigma_{\mathrm{N}} \nabla \mu$, respectively, with $e=-|e|$ and $\sigma_{\mathrm{N}}$ the conductivity of $\mathrm{N}$.

Equations (1) and (2) are complemented by BCs at the interfaces. At the interface with vacuum, one imposes a zero current condition, whereas at the I/N interface with ISOC, the BCs for the spin and charge densities read: ${ }^{27}$

$$
\begin{gathered}
-\left.\sigma_{\mathrm{N}}(\nabla \cdot \mathbf{n}) \hat{\mu}\right|_{0}=\left.G_{\perp} \hat{\mu}_{\perp}\right|_{0}+\left.G_{\|} \hat{\mu}_{\|}\right|_{0}+\left.\sigma_{\mathrm{cs}}(\mathbf{n} \times \nabla) \mu\right|_{0}, \\
-\left.\sigma_{\mathrm{N}}(\nabla \cdot \mathbf{n}) \mu\right|_{0}=\left.\sigma_{\mathrm{sc}}(\mathbf{n} \times \nabla) \hat{\mu}\right|_{0} .
\end{gathered}
$$

Here, $\mathbf{n}$ is the unitary vector normal to the interface, see Fig. 1(a). The last term in the rhs of Eq. (3) describes the charge-to-spin conversion quantified by the conductivity $\sigma_{\mathrm{cs}}$. This term couples an effective electric field and the (outgoing) spin current density at the interface ${ }^{27,29-31}$ and can be interpreted as an interfacial SHE. Alternatively, it can be interpreted as if the electric field induces a homogeneous spin ECP via an interfacial EE, which in turn diffuses into N. Both interpretations are fully compatible within the present formalism. The second type of process taking place at the interface are spin-losses [first two terms in the rhs of Eq. (3)], quantified by the spin-loss conductances per area $G_{\perp / \|}$ for spins perpendicular/parallel $\left(\hat{\mu}_{\perp} / \hat{\mu}_{\|}\right)$to the interface.

The charge is obviously conserved and, therefore, the rhs of Eq. (4) only contains the spin-to-charge conversion term. The latter is the reciprocal of the last term in Eq. (3) ${ }^{32}$ and can be interpreted as an interfacial inverse SHE but, again, an alternative interpretation is possible: from the conservation of the charge current at the interface, we can relate the bulk charge current to the divergence of an interfacial current $\mathbf{j}_{\mathrm{I}}$ as $\left.\sigma_{\mathrm{N}}(\nabla \cdot \mathbf{n}) \mu\right|_{0}=-e \nabla \cdot \mathbf{j}_{\mathrm{I}}$. Comparing the latter with Eq. (4), we define $\mathbf{j}_{\mathrm{I}}$ as ${ }^{33}$

$$
e \mathbf{j}_{\mathrm{I}}=-\left.\sigma_{\mathrm{sc}}(\mathbf{n} \times \hat{\mu})\right|_{0} .
$$

Written in this way, Eq. (4) describes the conversion of a nonequilibrium spin into an interfacial charge current, which corresponds to an interfacial IEE, see Fig. 1(b). This interpretation allows us to introduce the commonly used conversion length $\lambda_{\text {IEE }}$, defined as the ratio between the amplitude of the induced interfacial charge current density, $\mathbf{j}_{\mathrm{I}}$, and the amplitude of the spin current injected from the bulk, $\left.\sigma_{\mathrm{N}}(\nabla \cdot \mathbf{n}) \hat{\mu}\right|_{0}$. According to Eq. (5), the effect is finite only if the spin current is polarized in a direction parallel to the interface. Using Eqs. (3) and (5), we obtain

$$
\lambda_{\mathrm{IEE}}=\frac{\sigma_{\mathrm{sc}}}{G_{\|}} .
$$

This is a remarkable result that follows straightforwardly from our description of hybrid systems with ISOC and for which the spincharge interconversion occurs only at the interface. $\lambda_{\text {IEE }}$ is purely determined by interfacial parameters and it is indeed a quantification of the conversion efficiency: it is the ratio between the spin-to-charge conversion and the spin-loss at the interface. Both parameters, $\sigma_{\mathrm{sc}}$ and $G_{\|}$, depend on the microscopic properties of the interface, which are intrinsic for each material combination, and may depend on temperature.

From an experimental perspective, the spin-to-charge conversion is usually detected electrically, by measuring a voltage drop [see 


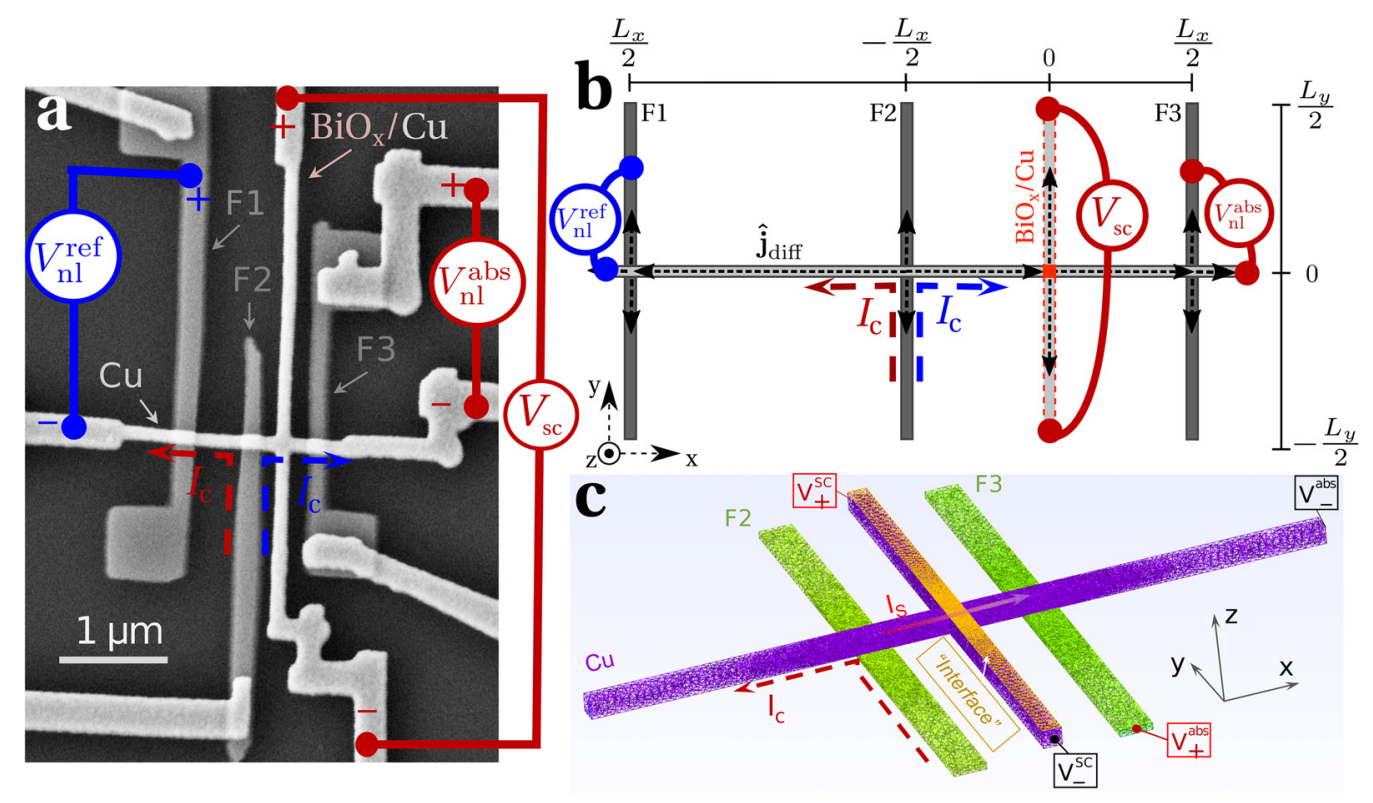

FIG. 2. (a) SEM image of the two Py/Cu LSVs, the reference one between ferromagnets F1-F2 and the one with a middle BiO $/$ /Cu wire (light red covering) between F2-F3. Non-local voltages $V_{\mathrm{nl}}^{\text {ref }}$ (blue circuit) and $V_{\mathrm{nl}}^{\text {abs }}$ (red circuit) are measured applying an external magnetic field $(B)$ along the $y$ axis. The spin-to-charge conversion voltage $V_{\mathrm{sc}}$ (red circuit) is detected applying $B$ along the $x$ axis. (b) Effective one-dimensional model of the device. (c) Geometry and mesh of the 3D finite element method model. The $\mathrm{BiO}_{x} / \mathrm{Cu}$ interface is simulated as a thin layer (yellow) on top of the transverse Cu wire (purple).

Figs. 2(a) and 4(a)]. For concreteness, we consider the generic setup of Fig. 1(b): a spin current polarized in the $x$ direction flows towards the interface, and a voltage difference is generated in the transverse $y$ direction according to Eq. (5). The averaged voltage drop between the points $y= \pm L_{y} / 2$ is given by (see supplementary material Note S1)

$$
V_{\mathrm{sc}}=\frac{\sigma_{\mathrm{sc}}}{e \sigma_{\mathrm{N}} A_{\mathrm{N}}} \iint_{-\frac{L_{y}}{2}}^{\frac{L_{y}}{2}}\left(\mathbf{n} \times\left.\hat{\mu}\right|_{0}\right) \cdot \mathbf{e}_{y} d x d y,
$$

where $\mathbf{e}_{y}$ is a unitary vector in the $y$ direction and $A_{\mathrm{N}}=t_{\mathrm{N}} w_{\mathrm{N}}$ is the wire cross section, with $t_{\mathrm{N}}$ and $w_{\mathrm{N}}$ being its thickness and width, over which the voltage drop is averaged. According to Eq. (7), the voltage drop between two points is proportional to the spin accumulation between them created via the ISOC. Next, we calculate the voltage drop associated with SCI in two different devices with an I/N interface.

We start analyzing the double Py/Cu LSV shown in Fig. 2(a) (see supplementary material Note S2 for experimental details). A charge current $I_{\mathrm{c}}$ is injected from the ferromagnetic injector F2 into the $\mathrm{Cu}$ wire. F2 forms a LSV either with the detector F1 or F3. We use the F1-F2 LSV as a reference device. In the F2-F3 LSV, there is an additional middle $\mathrm{Cu}$ wire covered by a $\mathrm{BiO}_{x}$ layer, resulting in an $\mathrm{I} / \mathrm{N}$ interface with ISOC, in which part of the spin current is absorbed and converted to a transverse charge current.

Quantitative description of electronic transport in LSVs has been widely studied in the literature. ${ }^{34,35}$ In our $\mathrm{Cu}$ wires, $\lambda_{\mathrm{N}} \gg t_{\mathrm{N}}, w_{\mathrm{N}}$, allowing us to simplify the ECPs diffusion to a one-dimensional problem, ${ }^{34-36}$ see Fig. 2(b) and supplementary material Note S3. At the $\mathrm{BiO}_{x} / \mathrm{Cu}$ wire, the $z$-integration using Eq. (3) leads to a renormalization of $\lambda_{\mathrm{N}}$,

$$
\lambda_{\mathrm{N} \|}=\frac{\lambda_{\mathrm{N}}}{\sqrt{1+\frac{G_{\|} \lambda_{\mathrm{N}}^{2}}{\sigma_{\mathrm{N}} t_{\mathrm{N}}}}} .
$$

At the node, $x=0$ in Fig. 2(b), we use Kirchhoffs law for the spin currents (see supplementary material Note S3),

$$
-\left.A_{\mathrm{N}} \sigma_{\mathrm{N}} \partial_{x} \hat{\mu}_{\|}\right|_{0^{-}} ^{0^{+}}=-\left.G_{\mathrm{N} \|} \hat{\mu}_{\|}\right|_{x=0}-A_{\mathrm{n}}^{\mathrm{eff}} \sigma_{\mathrm{cs}} \frac{e j_{\mathrm{c}}}{\sigma_{\mathrm{N}}} \hat{\mathbf{e}}_{x} .
$$

Here, $G_{\mathrm{N} \|}=\frac{t_{\mathrm{N}} \sigma_{\mathrm{N}} A_{\mathrm{n}}^{\text {eff }}}{\lambda_{\mathrm{N} \|}^{2}}$ is the effective spin (bulk) conductance of the $\mathrm{BiO}_{x} / \mathrm{Cu}$ wire, with $A_{\mathrm{n}}^{\text {eff }}=w_{\mathrm{N}}\left(w_{\mathrm{N}}+2 \lambda_{\mathrm{N} \|}\right)$. The latter is the effective area of the $\mathrm{BiO}_{x} / \mathrm{Cu}$ interface that absorbs (injects) spin current. Indeed, the rhs of this equation corresponds to Eq. (3) with an effective spin-loss conductance counting for both the interfacial and bulk spinlosses at the middle wire. The last term in Eq. (9) corresponds to the last term in Eq. (3) and it is proportional to the total injected charge current $I_{\mathrm{c}}$ along the middle wire oriented in the $y$ direction. If we assume an homogeneously distribution of the current, then $\mathbf{j}_{\mathrm{c}}=j_{\mathrm{c}} \hat{\mathbf{e}}_{y}$, with $j_{\mathrm{c}}=\frac{I_{\mathrm{c}}}{A_{\mathrm{N}}}$.

The $\mathrm{Cu} / \mathrm{Py}$ interfaces are described by the following $\mathrm{BC}:^{34,37,38}$

$$
\begin{gathered}
-\left.A_{\mathrm{N}} \sigma_{\mathrm{N}} \partial_{x} \hat{\mu}_{\|}\right|_{x=-\frac{L_{x}^{+}}{2}} ^{x=-\frac{L_{x}^{-}}{2}}=-A_{\mathrm{F}}\left(\left.\sigma_{\mathrm{F}}^{*} \partial_{y} \hat{\mu}_{\| \mathrm{F} 2}\right|_{y=0}+p_{\mathrm{F}} e j_{\mathrm{c}}\right), \\
-\left.A_{\mathrm{N}} \sigma_{\mathrm{N}} \partial_{x} \hat{\mu}_{\|}\right|_{x=\frac{L_{x}^{+}}{2}} ^{x=-L_{x}^{-}}=-\left.A_{\mathrm{F}} \sigma_{\mathrm{F}}^{*} \partial_{y} \hat{\mu}_{\| \mathrm{F} 3}\right|_{y=0},
\end{gathered}
$$

where $\hat{\mu}_{\| \mathrm{F} 2 / \mathrm{F} 3}$ is the spin ECP at F2/F3, $p_{\mathrm{F}}$ the spin polarization, and $\sigma_{\mathrm{F}}^{*}=\sigma_{\mathrm{F}}\left(1-p_{\mathrm{F}}^{2}\right)$ the effective conductivity of Py. $L_{x}$ is the distance 

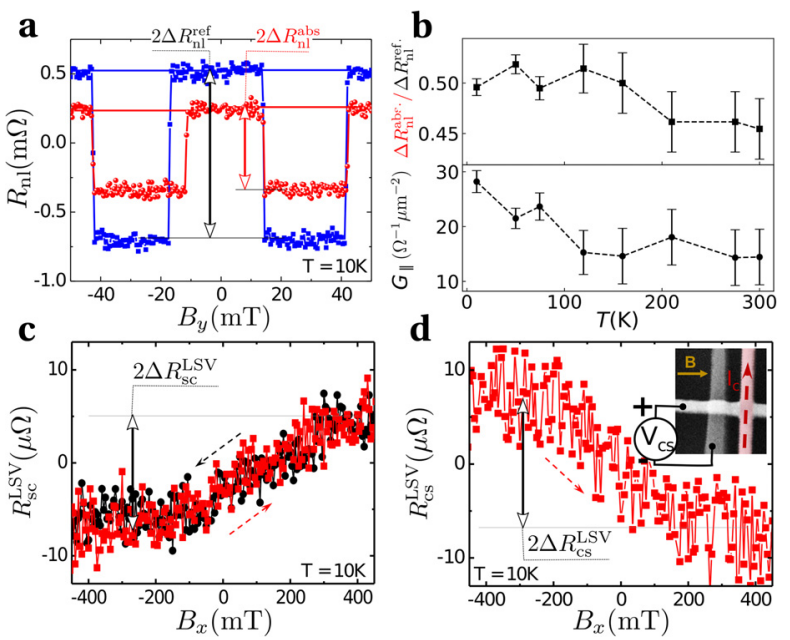

FIG. 3. (a) Non-local resistances as a function of $B_{y}$ (trace and retrace) measured at $I_{\mathrm{c}}=70 \mu \mathrm{A}$ and $10 \mathrm{~K}$ for the reference LSV (blue squares) and the $\mathrm{BiO}_{x} / \mathrm{Cu} \mathrm{LSV}$ (red circles). $\Delta R_{n}^{\text {ref }}$ and $\Delta R_{n !}^{\text {abs }}$ are tagged. (b) Temperature dependence of the spin absorption ratio (upper panel) and the corresponding spin-loss conductance (lower panel). (c) and (d) Reciprocal SCI non-local resistances as a function of $B_{x}$ (trace in red and retrace in black), from which we extract the spin-to-charge $\left(2 \Delta R_{\mathrm{sc}}^{\mathrm{LSV}}\right)$ from an average of seven sweeps and charge-to-spin $\left(2 \Delta R_{\mathrm{cS}}^{\mathrm{SS}}\right)$ signals from an average of four sweeps, respectively. Measurements are performed at $10 \mathrm{~K}$ and $I_{c}=70 \mu \mathrm{A}$ (c) and $I_{\mathrm{c}}=150 \mu \mathrm{A}$ (d).

between consecutive ferromagnetic wires and $A_{\mathrm{F}}$ the $\mathrm{Py} / \mathrm{Cu}$ junction area. ${ }^{34}$ For the reference LSV, we substitute F3 by F1 in Eq. (10). Because the $\mathrm{Py} / \mathrm{Cu}$ interfaces are electrically transparent, we assume the continuity of $\hat{\mu}_{\|}$. This condition, together with the onedimensional version of Eq. (1) and the BCs (9) and (10), determines the full spatial dependence of $\hat{\mu}_{\|}$.

Specifically, we need $\hat{\mu}_{\|}$at the detector F1/F3 to determine the non-local voltage $V_{\mathrm{nl}}=\left.e^{-1} p_{\mathrm{F}} \hat{\mu}_{\| \mathrm{F} 1 / \mathrm{F} 3}\right|_{y=0}{ }^{34,39}$ [see Fig. 2(b)] and the corresponding non-local resistance, $R_{\mathrm{nl}}=V_{\mathrm{nl}} / I_{\mathrm{c}}$, where $I_{\mathrm{c}}$ is the current injected from F2. $R_{\mathrm{nl}}$ changes sign when the magnetic configuration of the ferromagnetic injector and detector changes from parallel, $R_{\mathrm{nl}}^{\mathrm{P}}$, to antiparallel, $R_{\mathrm{nl}}^{\mathrm{AP}}$. This allows us to remove any baseline resistance coming from non-spin related effects by taking $\Delta R_{\mathrm{nl}}=R_{\mathrm{nl}}^{\mathrm{P}}-R_{\mathrm{nl}}^{\mathrm{AP}}$ [see Fig. 3(a)]. Comparing the resistance measured at F3, $\Delta R_{\mathrm{nl}}^{\mathrm{abs}}$, with the one measured at F1, $\Delta R_{\mathrm{nl}}^{\mathrm{ref}}$, we determine the magnitude of the spin absorption and, therefore, the value of the spin-loss conductance, $G_{\|}$. For this, we compute the ratio $\Delta R_{\mathrm{nl}}^{\mathrm{abs}} / \Delta R_{\mathrm{nl}}^{\mathrm{ref}}=\hat{\mu}_{\| \mathrm{F} 3} /\left.\hat{\mu}_{\| \mathrm{F} 1}\right|_{y=0}$ by solving the full boundary problem,

$$
\frac{\Delta R_{\mathrm{n}}^{\mathrm{abs}}}{\Delta R_{\mathrm{nl}}^{\mathrm{ref}}}=\left[1+\frac{G_{\mathrm{N} \|}}{2 G_{\mathrm{N}}} \frac{\left(G_{\mathrm{F}}+2 G_{\mathrm{N}}\right)-G_{\mathrm{F}} e^{-\frac{L_{x}}{L_{\mathrm{N}}}}}{\left(G_{\mathrm{F}}+2 G_{\mathrm{N}}\right)+G_{\mathrm{F}} e^{-\frac{L_{x}}{L_{\mathrm{N}}}}}\right]^{-1} .
$$

Here, $G_{i}=\frac{\sigma_{i} A_{i}}{\lambda_{i}}$ are the bare $\mathrm{Cu}(i=\mathrm{N})$ and $\mathrm{Py}(i=\mathrm{F})$ wires spin conductances. The form of Eq. (11) agrees with the one obtained in previous works. ${ }^{35,38,40}$ However, our expression is more general since it distinguishes via $G_{\mathrm{N} \|}$ between interfacial and bulk losses at the $\mathrm{BiO}_{x} /$ $\mathrm{Cu}$ wire. Consequently, we can ensure that our calculation of $G_{\|}$and, therefore, $\lambda_{\text {IEE }}$, is only related to interfacial effects [see Eqs. (6), (8), and (9)].

Figure 3(b) shows a weak temperature dependence of the absorption ratio, $\Delta R_{\mathrm{nl}}^{\text {abs }} / \Delta R_{\mathrm{nl}}^{\text {ref }} \approx 0.5$, revealing that about half of the spin current is absorbed at the $\mathrm{BiO}_{x} / \mathrm{Cu}$ middle wire. The temperature dependence of $\sigma_{\mathrm{N}}$ is measured (supplementary material Note S4), with $t_{\mathrm{N}}=w_{\mathrm{N}}=80 \mathrm{~nm}$ and $L_{x}=570 \mathrm{~nm}$. The specific properties of the Py and $\mathrm{Cu}$ wires $\left(\rho_{\mathrm{F}}\right.$ and $p_{\mathrm{F}}$ temperature dependencies and constant spin resistivities $\lambda_{\mathrm{F}} / \sigma_{\mathrm{F}}=0.91 \mathrm{f} \Omega \mathrm{m}^{2}$ and $\lambda_{\mathrm{N}} / \sigma_{\mathrm{N}}=18.3 \mathrm{f} \Omega \mathrm{m}^{2}$ ) are well characterized from our previous work. ${ }^{41}$ Thereupon, by inserting these experimental values into Eq. (11) for different temperatures, we obtain the $G_{\|}$dependence shown in Fig. 3(b). A slight decrease in $G_{\|}$can be observed with increasing temperature, which seems to arise from the $\mathrm{Cu}$ conductivity. A linear relation between $G_{\|}$and $\sigma_{\mathrm{N}}$ (see supplementary material Note S5A) suggests a Dyakonov-Perel mechanism of the spin-loss, expected for a Rashba interface and in agreement with Ref. 42.

We can also determine $\sigma_{\mathrm{sc} / \mathrm{cs}}$ in the same device. By injecting a charge current $I_{\mathrm{c}}$ from $\mathrm{F} 2$, an $x$-polarized spin current is created and reaches the $\mathrm{BiO}_{x} / \mathrm{Cu}$ wire, where a conversion to a transverse charge current occurs via Eq. (5). This is detected as a non-local voltage $V_{\mathrm{sc}}$ along the $\mathrm{BiO}_{x} / \mathrm{Cu}$ wire, determining the non-local resistance $R_{\mathrm{sc}}^{\mathrm{LSV}}$ $=V_{\mathrm{sc}} / I_{\mathrm{c}}$ as a function of an in-plane magnetic field $B_{x}$. By reversing the orientation of the magnetic field, the opposite $R_{\mathrm{sc}}^{\mathrm{LSV}}$ is obtained. The difference of the opposite values of $R_{\mathrm{sc}}^{\mathrm{LSV}}, 2 \Delta R_{\mathrm{sc}}^{\mathrm{LSC}}$ in Fig. 3(c), allows us to remove any baseline resistance. By swapping the voltage
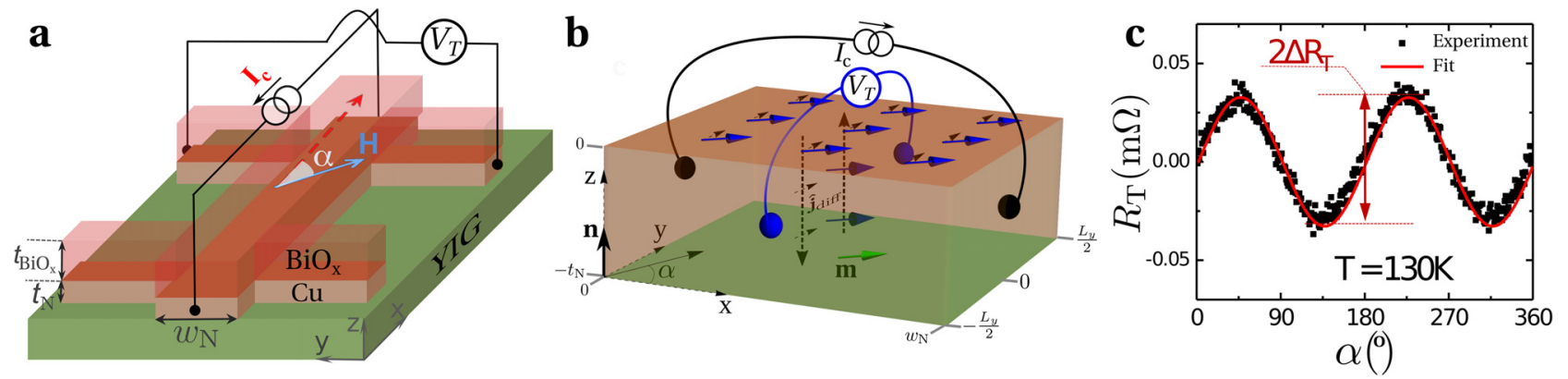

FIG. 4. (a) Measurement configuration of the TADMR in the $\mathrm{BiO}_{x} / \mathrm{Cu}$ Hall-cross device on YIG. An in-plane B-field $(100 \mathrm{mT})$ is rotated an angle $(\alpha)$ with respect to the applied current $\left(I_{c}=5 \mathrm{~mA}\right)$ direction. (b) Double $\mathrm{SCl}$ at the $\mathrm{BiO}_{x} / \mathrm{Cu}$ interface. (c) Transverse resistance measured as a function of $\alpha$ (black squares). The solid red curve corresponds to a fit to Eq. (14). 
and current probes, the reciprocal charge-to-spin conversion signal, $R_{\mathrm{cS}}^{\mathrm{LSV}}=V_{\mathrm{cs}} / I_{\mathrm{c}}$, can be determined.

Theoretically, from the full spatial dependence of $\hat{\mu}_{\|}$, we compute $V_{\mathrm{sc}}$ from Eq. (7) and $V_{\mathrm{cs}}$ from $V_{\mathrm{cs}}=\left.e^{-1} p_{\mathrm{F}} \mu_{\| \mathrm{F} 2}^{x}\right|_{y=0}$, yielding

$$
\Delta R_{\mathrm{sc} / \mathrm{cs}}^{\mathrm{LSV}}= \pm \frac{\sigma_{\mathrm{sc} / \mathrm{cs}}}{\sigma_{\mathrm{N}}} \frac{A_{\mathrm{n}}^{\mathrm{eff}}}{A_{\mathrm{N}}} \frac{p_{\mathrm{F}} e^{\frac{L_{x}}{2 \lambda_{\mathrm{N}}}}}{G_{\mathrm{F}}\left(1-\frac{G_{\mathrm{N} \|}}{2 G_{\mathrm{N}}}\right)+e^{\frac{L_{x}}{\lambda_{\mathrm{N}}}}\left(G_{\mathrm{F}}+2 G_{\mathrm{N}}\right)\left(1+\frac{G_{\mathrm{N} \|}}{2 G_{\mathrm{N}}}\right)} .
$$

Experimentally, Figs. 3(c) and 3(d) confirm the reciprocity between both measurements, $\Delta R_{\mathrm{cs}}^{\mathrm{LSV}}=\Delta R_{\mathrm{sc}}^{\mathrm{LSV}}$. The broken time reversal symmetry, due to the magnetic contacts, leads to the opposite sign for reciprocal measurements. Contrasting this with the result of Eq. (12), one confirms that $\sigma_{\mathrm{sc}}=\sigma_{\mathrm{cs}}{ }^{43}$ The experimental value, $2 \Delta R_{\mathrm{sc}}^{\mathrm{LSV}}$ $\approx 15 \pm 3 \mu \Omega$ at $10 \mathrm{~K}$, yields $\sigma_{\mathrm{sc} / \mathrm{cs}} \approx 44 \pm 9 \Omega^{-1} \mathrm{~cm}^{-1}$ and $\lambda_{\mathrm{IEE}}$ $\approx 0.16 \pm 0.03 \mathrm{~nm}$. The latter value is of the same order of magnitude but somewhat smaller than the previously reported results obtained by spin pumping experiments, $\lambda_{\text {IEE }} \approx 0.2-0.7 \mathrm{~nm},{ }^{15,18,42}$ and LSV experiments, $\lambda_{\text {IEE }} \approx 0.5-1 \mathrm{~nm}^{36}$ This discrepancy might be due to a different quality of the $\mathrm{BiO}_{x} / \mathrm{Cu}$ interface: ex situ deposition in our experiment and in situ deposition in other works. The temperature dependence of the different parameters is presented in Note S5B in the supplementary material. One observes a decreasing trend of $\sigma_{\text {sc }}$ by increasing the temperature, which translates in a decrease of $\lambda_{\mathrm{IEE}}$, in agreement with the previous literature. ${ }^{42}$

The accuracy of our 1D model is checked by performing a 3D finite element method simulation detailed in supplementary material Note S6. Figure 2(c) shows the geometry of the simulated device and the mesh of the finite elements. The ISOC is simulated as a thin layer of finite thickness $t_{\text {int }}$, spin diffusion length $\lambda_{\text {int }}$, and a spin Hall angle $\theta_{\text {int }}^{\text {eff }}$. From the definition $\lambda_{\text {IEE }}=\frac{1}{2} \theta_{\text {int }}^{\text {eff }} t_{\text {int }}$, we obtain $\lambda_{\text {IEE }}=0.10 \pm 0.02 \mathrm{~nm}$, in good agreement with our $1 \mathrm{D}$ model.

To verify that both ISOC parameters, $G_{\|}$and $\sigma_{\mathrm{sc}}$, are interface specific, we carry out another experiment involving a $\mathrm{BiO}_{x} / \mathrm{Cu}$ interface. Namely, we measure the SMR in a Cu layer sandwiched between $\mathrm{BiO}_{x}$ (at $z=0$ ) and $\mathrm{Y}_{3} \mathrm{Fe}_{5} \mathrm{O}_{12}$ (YIG) insulating layers (at $z=-t_{\mathrm{N}}$ ), shaped as a Hall bar, see Fig. 4(a) and supplementary material Note S2 for the experimental details. In this setup, a double SCI takes place as sketched in Fig. 4(b). A charge current $I_{\mathrm{c}}$ in the $x$ direction induces an out-of-plane $y$-polarized spin current density via Eq. (3). This spin current propagates towards the $\mathrm{Cu} / \mathrm{YIG}$ interface where it is partly reflected with mixed $x$ and $y$ polarizations. ${ }^{45-47}$ The reflected spin current diffuses back to the $\mathrm{BiO}_{x} / \mathrm{Cu}$ interface, where its $x$-polarized contribution is reciprocally converted to an interfacial charge current. The overall effect is then proportional to $\sigma_{\mathrm{cs}} \sigma_{\mathrm{sc}}=\sigma_{\mathrm{sc}}^{2}$.

The electron spin reflection at the $\mathrm{Cu} / \mathrm{YIG}$ interface depends on the direction of magnetization $\mathbf{m}$ of ferrimagnetic YIG. The effective $\mathrm{BC}$ describing this interface is known and reads ${ }^{48,49}$

$$
\begin{aligned}
-\left.\sigma_{\mathrm{N}}(\nabla \cdot \mathbf{n}) \hat{\mu}\right|_{-t_{\mathrm{N}}}= & \left.G_{\mathrm{s}} \hat{\mu}\right|_{-t_{\mathrm{N}}}+G_{\mathrm{r}} \mathbf{m} \times\left.(\hat{\mu} \times \mathbf{m})\right|_{-t_{\mathrm{N}}} \\
& +\left.G_{\mathrm{i}}(\mathbf{m} \times \hat{\mu})\right|_{-t_{\mathrm{N}}} .
\end{aligned}
$$

Here, $G_{\mathrm{s}}$ is the so-called spin-sink conductance and $G_{\mathrm{r}, \mathrm{i}}$ are the real and imaginary parts of the spin-mixing conductance (per area), $G_{\uparrow \downarrow}=G_{\mathrm{r}}+i G_{\mathrm{i}}$. In YIG, $G_{\mathrm{i}} \ll G_{\mathrm{r}}$ and, hence, $G_{\mathrm{i}}$ is neglected. ${ }^{47,50-52}$

We measure the transverse angular dependent magnetoresistance (TADMR) in the $\mathrm{BiO}_{x} / \mathrm{Cu} / \mathrm{YIG}$ Hall bar of Fig. 4(a). The transverse voltage, $V_{\mathrm{T}}$, depends on the direction of the in-plane applied magnetic field, parameterized by the angle $\alpha$. The TADMR measurements are shown in Fig. 4(c).

Theoretically, we calculate the spatial dependence of $\hat{\mu}$ by solving the boundary problem of Eqs. (1), (3), and (13) by assuming translational invariance in the $x-y$ plane. We then determine $V_{\mathrm{T}}$ from Eq. (7) and obtain for $R_{\mathrm{T}}=V_{\mathrm{T}} / I_{\mathrm{c}}$,

$$
R_{\mathrm{T}} \approx \frac{\sigma_{\mathrm{sc}}^{2}}{2 \sigma_{\mathrm{N}}^{2} t_{\mathrm{N}}^{2}} \frac{G_{\mathrm{r}}}{\left(G_{\|}+G_{\mathrm{s}}\right)\left(G_{\|}+G_{\mathrm{s}}+G_{\mathrm{r}}\right)} \sin (2 \alpha)=\Delta R_{\mathrm{T}} \sin (2 \alpha) .
$$

Here, $\Delta R_{\mathrm{T}}$ is the amplitude of the modulation and we assume that $\lambda_{\mathrm{N}} \gg t_{\mathrm{N}}$ (see supplementary material Note S7). The parameters of the $\mathrm{Cu} / \mathrm{YIG}$ interface, $G_{\mathrm{r}, \mathrm{s}}$, add to the spin-loss at the $\mathrm{BiO}_{x} / \mathrm{Cu}$ interface $G_{\|}$. We identify by comparison of Eqs. (3) and (13) two effective spinloss conductances, $G_{x}=\left(G_{\|}+G_{\mathrm{s}}\right)$ and $G_{y}=\left(G_{\|}+G_{\mathrm{s}}+G_{\mathrm{r}}\right)$, for spins polarized in the $x$ and $y$ directions, respectively. The amplitude of the SMR signal, Eq. (14), is then proportional to $G_{x}-G_{y}$.

From Fig. 4(c), we estimate $\Delta R_{\mathrm{T}} \approx 0.03 \mathrm{~m} \Omega$ at $T=130 \mathrm{~K}$. At this temperature, from the LSV measurements, we obtain $G_{\|} \approx 1.5$ $\times 10^{13} \Omega^{-1} \mathrm{~m}^{-2}$ and $\sigma_{\mathrm{sc} / \mathrm{cs}} \approx 11.3 \Omega^{-1} \mathrm{~cm}^{-1}$, as shown in Figs. 3(b) and S3b, respectively. The spin conductances $G_{\mathrm{s}}$ and $G_{\mathrm{r}}$ in light metal/ YIG interfaces have been estimated in evaporated $\mathrm{Cu}^{53}$ and $\mathrm{Al}^{54}$ Whereas $G_{s}=3.6 \times 10^{12} \Omega^{-1} \mathrm{~m}^{-2}$ for $\mathrm{Cu} / \mathrm{YIG}^{53}$ is a consistent value in the literature, ${ }^{54,55}$ the reported $G_{\mathrm{r}}$ is very low, ${ }^{53}$ as generally observed in evaporated metals on YIG. ${ }^{54,56}$ By substituting $G_{\|}, G_{s}$, and $\sigma_{\mathrm{sc} / \mathrm{cs}}$ values in Eq. (14), we obtain $G_{\mathrm{r}} \approx 6.1 \times 10^{13} \Omega^{-1} \mathrm{~m}^{-2}$. This value for sputtered $\mathrm{Cu}$ on YIG is much larger than that estimated in evaporated $\mathrm{Cu}$ on YIG, in agreement with the reported difference between sputtered and evaporated Pt. ${ }^{56}$ Importantly, the obtained $G_{\mathrm{r}}$ satisfies the required condition $G_{s}<G_{\mathrm{r}},{ }^{47,55}$ which confirms the validity of our estimation.

In summary, we present a complete theoretical framework based on the drift-diffusion equations to accurately describe electronic transport in systems with ISOC at non-magnetic metal/insulator interfaces. Within our model, the interface is described by two types of processes: spin-losses, parameterized by the interfacial conductances $G_{\| / \perp}$, and SCI, quantified by $\sigma_{\text {sc }}$ and $\sigma_{\mathrm{cs}}$. These parameters are material specific. The efficiency of the spin-to-charge conversion is quantified by the ratio $\sigma_{\mathrm{sc}} / G_{\|}$, which coincides with the commonly used Edelstein length $\lambda_{\text {IEE }}$. The Onsager reciprocity ${ }^{57-59}$ is directly captured by $\sigma_{\mathrm{sc}}=\sigma_{\mathrm{cs}}$, as demonstrated by comparing our theoretical and experimental results. Our theory is an effective tool for an accurate quantification of SCI phenomena at interfaces, which is of paramount importance in many spintronic devices. It is important to emphasize that the present formulation of our theory is valid for interfaces between non-magnetic materials. In principle, one could go beyond our theory and address the problem of magnetic moment transfer at a metal/magnetic insulator interface by including interfacial exchange interaction and magnon dynamics into the model. ${ }^{47}$

See the supplementary material for additional details on the derivation of the spin-to-charge averaged voltage, Eq. (7), and the renormalized spin diffusion length and node boundary condition for the LSV, Eqs. (8) and (9), respectively; measured temperature dependence of the $\mathrm{Cu}$ resistivity and analysis on the temperature dependence of 
the ISOC parameters in the LSV; a brief explanation of the 3D simulation and the relation between the simulation and ISOC parameters; theoretical result for the transverse resistance measured in the multilayer Hall bar, i.e., which leads to Eq. (14); and the experimental details of the nanofabrication and measurements of the LSV and multilayer Hall bar devices.

\section{AUTHORS' CONTRIBUTIONS}

C.S-F. and V.T.P. contributed equally to this work.

C.S-F., F.S.B., and I.V.T. acknowledge funding from the Spanish Ministerio de Ciencia, Innovación y Universidades (MICINN) (Project Nos. FIS2016-79464-P and FIS2017-82804-P) and Grupos Consolidados UPV/EHU del Gobierno Vasco (Grant No. IT1249-19). The work of F.S.B. is partially funded by EU's Horizon 2020 research and innovation program under Grant Agreement No. 800923 (SUPERTED). The work at nanoGUNE is supported by Intel Corporation through the Semiconductor Research Corporation under MSR-INTEL TASK No. 2017-IN-2744 and the "FEINMAN" Intel Science Technology Center and by the Spanish MICINN under the Maria de Maeztu Units of Excellence Programme (No. MDM-2016-0618) and under Project Nos. MAT2015-65159-R and RTI2018-094861-B-100. V.T.P. acknowledges postdoctoral fellowship support from the "Juan de la Cierva-Formación” program by the Spanish MICINN (Grant No. FJCI-2017-34494). E.S. thanks the Spanish MECD for a Ph.D. fellowship (Grant No. FPU14/03102).

\section{DATA AVAILABILITY}

The data that support the findings of this study are available from the corresponding authors upon reasonable request.

\section{REFERENCES}

1'I. Žutić, J. Fabian, and S. D. Sarma, Rev. Mod. Phys. 76, 323 (2004).

${ }^{2}$ G. Vignale and J. Supercond, Nov. Magn. 23, 3 (2010).

${ }^{3}$ J. Sinova, S. O. Valenzuela, J. Wunderlich, C. H. Back, and T. Jungwirth, Rev. Mod. Phys. 87, 1213 (2015).

${ }^{4}$ S. O. Valenzuela and M. Tinkham, Nature 442, 176 (2006).

${ }^{5}$ T. Kimura, Y. Otani, T. Sato, S. Takahashi, and S. Maekawa, Phys. Rev. Lett. 98, 156601 (2007).

${ }^{6}$ A. Aronov and Y. B. Lyanda-Geller, JETP Lett. 50, 431 (1989), available at http://www.jetpletters.ac.ru/ps/1132/article_17140.shtml.

${ }^{7}$ V. M. Edelstein, Solid State Commun. 73, 233 (1990).

${ }^{8}$ Y. Ando and M. Shiraishi, J. Phys. Soc. Jpn. 86, 011001 (2017).

${ }^{9}$ A. Soumyanarayanan, N. Reyren, A. Fert, and C. Panagopoulos, Nature 539, 509 (2016).

${ }^{10}$ I. M. Miron, K. Garello, G. Gaudin, P.-J. Zermatten, M. V. Costache, S. Auffret, S. Bandiera, B. Rodmacq, A. Schuhl, and P. Gambardella, Nature 476, 189 (2011).

${ }^{11}$ L. Liu, C.-F. Pai, Y. Li, H. Tseng, D. Ralph, and R. Buhrman, Science 336, 555 (2012).

${ }^{12}$ C. Safeer, E. Jué, A. Lopez, L. Buda-Prejbeanu, S. Auffret, S. Pizzini, O. Boulle, I. M. Miron, and G. Gaudin, Nat. Nanotechnol. 11, 143 (2016).

${ }^{13}$ V. T. Pham, I. Groen, S. Manipatruni, W. Y. Choi, D. E. Nikonov, E. Sagasta, C.-C. Lin, T. A. Gosavi, A. Marty, L. E. Hueso, I. A. Young, and F. Casanova, Nat. Electron. 3, 309 (2020).

${ }^{14}$ S. Manipatruni, D. E. Nikonov, C.-C. Lin, T. A. Gosavi, H. Liu, B. Prasad, Y.-L. Huang, E. Bonturim, R. Ramesh, and I. A. Young, Nature 565, 35 (2019).

${ }^{15}$ S. Karube, K. Kondou, and Y. Otani, Appl. Phys. Express 9, 033001 (2016).

${ }^{16}$ J. Kim, Y.-T. Chen, S. Karube, S. Takahashi, K. Kondou, G. Tatara, and Y. Otani, Phys. Rev. B 96, 140409 (2017).
${ }^{17}$ H. Nakayama, Y. Kanno, H. An, T. Tashiro, S. Haku, A. Nomura, and K. Ando, Phys. Rev. Lett. 117, 116602 (2016).

${ }^{18}$ H. Tsai, S. Karube, K. Kondou, N. Yamaguchi, F. Ishii, and Y. Otani, Sci. Rep. 8, 5564 (2018).

${ }^{19}$ J.-C. Rojas-Sánchez, S. Oyarzún, Y. Fu, A. Marty, C. Vergnaud, S. Gambarelli, L. Vila, M. Jamet, Y. Ohtsubo, A. Taleb-Ibrahimi, P. Le Fèvre, F. Bertran, N. Reyren, J.-M. George, and A. Fert, Phys. Rev. Lett. 116, 096602 (2016).

${ }^{20}$ K. Kondou, R. Yoshimi, A. Tsukazaki, Y. Fukuma, J. Matsuno, K. Takahashi, M. Kawasaki, Y. Tokura, and Y. Otani, Nat. Phys. 12, 1027 (2016).

${ }^{21}$ E. Lesne, Y. Fu, S. Oyarzun, J. Rojas-Sánchez, D. Vaz, H. Naganuma, G. Sicoli, J.-P. Attané, M. Jamet, E. Jacquet et al., Nat. Mater. 15, 1261 (2016).

${ }^{22}$ D. C. Vaz, P. Noël, A. Johansson, B. Göbel, F. Y. Bruno, G. Singh, S. MckeownWalker, F. Trier, L. M. Vicente-Arche, A. Sander et al., Nat. Mater. 18, 1187 (2019).

${ }^{23}$ Y. Niimi, Y. Kawanishi, D. H. Wei, C. Deranlot, H. X. Yang, M. Chshiev, T. Valet, A. Fert, and Y. Otani, Phys. Rev. Lett. 109, 156602 (2012).

${ }^{24}$ J. Linder and T. Yokoyama, Phys. Rev. Lett. 106, 237201 (2011).

${ }^{25}$ I. V. Tokatly, E. E. Krasovskii, and G. Vignale, Phys. Rev. B 91, 035403 (2015).

${ }^{26}$ J. Borge and I. V. Tokatly, Phys. Rev. B 96, 115445 (2017).

${ }^{27}$ J. Borge and I. V. Tokatly, Phys. Rev. B 99, 241401 (2019).

${ }^{28}$ C. Sanz-Fernández, J. Borge, I. V. Tokatly, and F. S. Bergeret, Phys. Rev. B 100, 195406 (2019).

${ }^{29}$ V. P. Amin, J. Zemen, and M. D. Stiles, Phys. Rev. Lett. 121, 136805 (2018).

${ }^{30}$ V. P. Amin and M. D. Stiles, Phys. Rev. B 94, 104420 (2016).

${ }^{31}$ V. P. Amin and M. D. Stiles, Phys. Rev. B 94, 104419 (2016).

${ }^{32}$ Symmetry arguments alone cannot fix the relation between $\sigma_{\mathrm{sc}}$ and $\sigma_{\mathrm{cs}}{ }^{27}$ However, we will see by contrasting theory with experiment, that reciprocity requires $\sigma_{\mathrm{sc}}=\sigma_{\mathrm{cs}}$.

${ }^{33}$ Notice that, in principle, an additional divergenceless term may appear in the rhs of Eq. (5). Indeed, as demonstrated in Ref. 27, symmetry allows for a term proportional to the out-of-plane component of the spin ECP. In the present work, we only consider spin polarization parallel to the I/N interface and, hence, we neglect that term.

${ }^{34}$ S. Takahashi and S. Maekawa, Phys. Rev. B 67, 052409 (2003).

${ }^{35}$ Y. Niimi, H. Suzuki, Y. Kawanishi, Y. Omori, T. Valet, A. Fert, and Y. Otani, Phys. Rev. B 89, 054401 (2014).

${ }^{36}$ H. Isshiki, P. Muduli, J. Kim, K. Kondou, and Y. Otani, preprint arXiv:1901.03095 (2019).

${ }^{37}$ V. T. Pham, L. Vila, G. Zahnd, A. Marty, W. Savero-Torres, M. Jamet, and J.-P. Attané, Nano Lett. 16, 6755 (2016).

${ }^{38}$ T. Kimura, J. Hamrle, and Y. Otani, Phys. Rev. B 72, 014461 (2005).

${ }^{39}$ T. Kimura, J. Hamrle, and Y. Otani, Appl. Phys. Lett. 85, 3501 (2004).

${ }^{40}$ M. Isasa, M. C. Martínez-Velarte, E. Villamor, C. Magén, L. Morellón, J. M. De Teresa, M. R. Ibarra, G. Vignale, E. V. Chulkov, E. E. Krasovskii, L. E. Hueso, and F. Casanova, Phys. Rev. B 93, 014420 (2016).

${ }^{41}$ E. Sagasta, Y. Omori, M. Isasa, Y. Otani, L. E. Hueso, and F. Casanova, Appl. Phys. Lett. 111, 082407 (2017).

${ }^{42}$ H. Tsai, K. Kondou, and Y. Otani, Jpn. J. Appl. Phys., Part 1 58, 110907 (2019).

${ }^{43}$ In principle, any transport measurement in a system with a linear response will fulfill the Onsager reciprocity. ${ }^{57-59}$ Within our theory, the global reciprocity is guarantied by $\sigma_{s c}=\sigma_{c s}$. Importantly, the global reciprocity does not imply a point-wise identity of the local current densities.

${ }^{44}$ J. R. Sánchez, L. Vila, G. Desfonds, S. Gambarelli, J. Attané, J. De Teresa, C. Magén, and A. Fert, Nat. Commun. 4, 2944 (2013).

${ }^{45}$ Y.-T. Chen, S. Takahashi, H. Nakayama, M. Althammer, S. T. B. Goennenwein, E. Saitoh, and G. E. W. Bauer, Phys. Rev. B 87, 144411 (2013).

${ }^{46}$ H. Nakayama, M. Althammer, Y.-T. Chen, K. Uchida, Y. Kajiwara, D. Kikuchi, T. Ohtani, S. Geprägs, M. Opel, S. Takahashi et al., Phys. Rev. Lett. 110, 206601 (2013).

${ }^{47}$ X.-P. Zhang, F. S. Bergeret, and V. N. Golovach, Nano Lett. 19, 6330 (2019).

${ }^{48}$ A. Brataas, Y. V. Nazarov, and G. E. Bauer, Phys. Rev. Lett. 84, 2481 (2000).

${ }^{49}$ Following our convention, the vector $\mathbf{n}$ normal to the interface, points from the $\mathrm{Cu}$ towards the insulating YIG layer.

${ }^{50}$ N. Vlietstra, J. Shan, V. Castel, J. Ben Youssef, G. E. W. Bauer, and B. J. van Wees, Appl. Phys. Lett. 103, 032401 (2013). 
${ }^{51}$ M. Althammer, S. Meyer, H. Nakayama, M. Schreier, S. Altmannshofer, M. Weiler, H. Huebl, S. Geprägs, M. Opel, R. Gross, D. Meier, C. Klewe, T. Kuschel, J.-M. Schmalhorst, G. Reiss, L. Shen, A. Gupta, Y.-T. Chen, G. E. W. Bauer, E. Saitoh, and S. T. B. Goennenwein, Phys. Rev. B 87, 224401 (2013).

${ }^{52}$ T. Kosub, S. Vélez, J. M. Gomez-Perez, L. E. Hueso, J. Fassbender, F. Casanova, and D. Makarov, Appl. Phys. Lett. 113, 222409 (2018).

${ }^{53}$ E. Villamor, M. Isasa, S. Vélez, A. Bedoya-Pinto, P. Vavassori, L. E. Hueso, F. S. Bergeret, and F. Casanova, Phys. Rev. B 91, 020403 (2015).

${ }^{54}$ K. S. Das, F. K. Dejene, B. J. van Wees, and I. J. Vera-Marun, Appl. Phys. Lett. 114, 072405 (2019).
${ }^{55}$ L. J. Cornelissen, K. J. H. Peters, G. E. W. Bauer, R. A. Duine, and B. J. van Wees, Phys. Rev. B 94, 014412 (2016).

${ }^{56} \mathrm{~N}$. Vlietstra, J. Shan, V. Castel, B. J. van Wees, and J. Ben Youssef, Phys. Rev. B 87, 184421 (2013).

${ }^{57}$ H. B. G. Casimir, "On Onsager's principle of microscopic reversibility," Rev. Mod. Phys. 17, 343-350 (1945).

${ }^{58} \mathrm{~L}$. Onsager, "Reciprocal relations in irreversible processes. I," Phys. Rev. 37, 405 (1931).

${ }^{59}$ L. Onsager, "Reciprocal relations in irreversible processes. II," Phys. Rev. 38, 2265 (1931). 